\title{
Nonlinear Disturbance Decoupling for a Nonholonomic Mobile Robotic Manipulation Platform
}

\author{
Joel Jimenez-Lozano and Bill Goodwine \\ Department of Aerospace and Mechanical Engineering \\ University of Notre Dame \\ Notre Dame, IN 46556, USA
}

\begin{abstract}
A mobile manipulator is at the present time a widespread term to refer to robot systems built from a robotic manipulator arm mounted on a mobile platform. A mobile manipulation system offers a dual advantage of mobility offered by a mobile platform and dexterity offered by the manipulator. In this work, the tracking and nonlinear disturbance decoupling problems are studied. We show that this system posses the necessary geometric structure for complete disturbance decoupling between the outputs and disturbances. Simulation results obtained for the mobile manipulator show good performance in the presence of significant disturbances using the designed nonlinear controller.
\end{abstract}

Index Terms-Mobile manipulator, nonlinear control, disturbance decoupling.

\section{INTRODUCTION}

In recent years, increasing attention has been paid to the control problems of robotic manipulators with more "complete" models and more dynamic effects taken into account for better performance. Mobile manipulators built from a robotic arm mounted on a wheeled mobile platform provides better capabilities for numerous tasks. A mobile manipulator combines the dextrous manipulation capability offered by the manipulator and the motility provided by the mobile platform. The integration of a manipulator and a mobile platform gives rise to many interesting issues. Investigations on their stability, control design, simulation and experimentation for different situations has been studied by a number of researchers [1][3].

Yamamoto and Yun [4] studied the effect of the dynamic interaction between the manipulator and the mobile platform on the task performance, and showed that the system was feedback linearizable under the appropriate nonlinear change of coordinates. The manipulator tracks a desired trajectory in a fixed reference frame. Their objective was to compensate the dynamic interaction through a nonlinear feedback to improve the performance of the overall system. The motion's equations of the mobile manipulator were obtained. A state space formulation was presented, motion equations and a constraint equation were considered. A nonlinear feedback controller was designed to compensate for the dynamic interaction. A modular approach of his analysis was presented in [5] which includes a detailed proof of the functional dependence of some of the dynamic terms of the equations. In this work, that methodology will be extended in studying a mobile manipulator in which we will include external force disturbances into the system.

As mentioned, the modeling and design of nonlinear disturbance decoupling control for the wheeled mobile manipulator is addressed. The final goal of the disturbance decoupling is to find a state feedback law such that the output is unaffected by the disturbance. These disturbances can be external forces on the system as environmental loads. Related work on disturbance decoupling have been studied on robotic manipulators and mobile platforms in [6], [7].

In the following sections, we will present the dynamic equations of the mobile manipulator which are coupled. A state space representation of the equations will be presented. A nonlinear feedback controller will be designed, which includes disturbance decoupling. Computer simulations will be displayed, results shown include the position of the mobile manipulator during motion to follow a task trajectory, heading angles, joint-angles of the manipulator, etc. It will be shown that the outputs are satisfactorily decoupled from the disturbances.

\section{Modeling EQuations}

The motion equation of the robotic manipulator subject to vehicle motion [4], [5] can be extended to include external force disturbances, and it is given by

$$
\begin{gathered}
M_{r}\left(q_{r}\right) \ddot{q}_{r}+C_{r 1}\left(q_{r}, \dot{q}_{r}\right)+C_{r 2}\left(q_{r}, \dot{q}_{r}, \dot{q}_{v}\right)=\tau_{r} \\
-R_{r}\left(q_{r}, q_{v}\right) \ddot{q}_{v}+J_{r}^{T}\left(q_{r}\right) F_{e}{ }^{r},
\end{gathered}
$$

where $q_{r}=\left[\theta_{1}, \theta_{2}\right]^{T}$ denotes the Lagrangian coordinates of the manipulator, $q_{v}$ denotes the Lagrangian coordinates of the mobile platform. $M_{r}$ is the inertia Matrix, $C_{r 1}$ represents the Coriolis and centrifugal terms, $C_{r 2}$ denotes the Coriolis and centrifugal terms caused by the angular motion of the platform. $\tau_{r}$ is the input torque/force for the manipulator. $R_{r}$ is the inertia matrix which represents the effect of the vehicle dynamics on the manipulator. $F_{e}{ }^{r}$ is an end-effector external force disturbance vector on the manipulator and $J_{r}^{T}\left(q_{r}\right)$ is the task space Jacobian matrix for the manipulator. Each term matrix/vector is presented in the Appendix. 
The motion equation of the mobile platform with a mounted manipulator [4], [5] including external force disturbances is given by

$$
\begin{gathered}
M_{v 1}\left(q_{v}\right) \ddot{q}_{v}+C_{v 1}\left(q_{v}, \dot{q}_{v}\right)+C_{v 2}\left(q_{r}, \dot{q}_{r}, q_{v}, \dot{q}_{v}\right)= \\
E_{v} \tau_{v}-A^{T} \lambda-M_{v 2}\left(q_{r}, q_{v}\right) \ddot{q}_{v}-R_{v}\left(q_{r}, q_{v}\right) \ddot{q}_{r} \\
+E_{v} J_{v}{ }^{T}\left(q_{v}\right) F_{e}{ }^{v},
\end{gathered}
$$

where $M_{v 1}$ and $C_{v 1}$ are the mass inertia and the velocity dependent terms of the platform, respectively. $M_{v 2}$ and $C_{v 2}$ represent the inertial term and Coriolis and centrifugal terms due to the presence of the manipulator. $\tau_{v}$ is the input torque/force to the vehicle. $E_{v}$ is a constant matrix, $\lambda$ denotes the vector Lagrange multipliers corresponding to the kinematic constraints, and $R_{v}$ represents the inertia matrix which reflects the dynamic effect of the arm motion on the vehicle. $F_{e}{ }^{v}$ is an external force disturbance vector on the mobile platform through its center and $J_{v}{ }^{T}\left(q_{v}\right)$ is the moving space Jacobian matrix for the mobile platform. Each term matrix/vector is presented in the Appendix.

Combining the velocity and inertia terms in Eq. 1 and Eq. 2 , respectively, the motion equations of the wheeled mobile manipulator are simplified to

$$
\begin{gathered}
M_{r}\left(q_{r}\right) \ddot{q}_{r}+C_{r}\left(q_{r}, \dot{q}_{r}, \dot{q}_{v}\right)=\tau_{r} \\
-R_{r}\left(q_{r}, q_{v}\right) \ddot{q}_{v}, \\
M_{v}\left(q_{r}, q_{v}\right) \ddot{q}_{v}+C_{v}\left(q_{r}, q_{v}, \dot{q}_{r}, \dot{q}_{v}\right)=E_{v} \tau_{v} \\
-R_{v}\left(q_{r}, q_{v}\right) \ddot{q}_{r}-A^{T} \lambda+J_{r}^{T}\left(q_{r}\right) F_{e}^{r} \\
+E_{v} J_{v}^{T}\left(q_{v}\right) F_{e}{ }^{v},
\end{gathered}
$$

where $C_{r}=C_{r 1}+C_{r 2}, C_{v}=C_{v 1}+C_{v 2}$ and $M_{v}=M_{v 1}+$ $M_{v 2}$.

\section{A. Constraint Equations of the Mobile Platform}

The following notations will be used in the derivation of the constraint and dynamic equations, they are illustrated in Fig. 1.

- For the mobile platform, $\left(x_{0}, y_{0}\right)$ are the coordinates of the point $P_{0}$ which is the intersection of the axis of symmetry with the driving wheel axis in the inertial frame. $b$ is the distance between the driving wheels and the axis of symmetry. $r$ is the radius of each driving wheel. $\theta_{r}$ and $\theta_{l}$ are the angular positions of the right and left driving wheel, respectively. In addition, $\phi=r\left(\theta_{r}-\theta_{l}\right) / 2 b=c\left(\theta_{r}-\theta_{l}\right)$ is the heading angle of the mobile robot measured from ${ }^{w} X$-axis. $d$ is the distance from $P_{0}$ to the center of mass of the platform. $m_{c}$ is the mass of the platform without the driving wheels. $I_{c}$ is the moment of inertia of the platform without the driving wheels about a vertical axis through $P_{0}$.

- For the manipulator, where $P_{b}=\left({ }^{v} x_{b},{ }^{v} y_{b}\right)$ are the coordinates of the base of the manipulator in the frame $\Sigma_{v} . \theta_{1}$ and $\theta_{2}$ are the joint angles of the manipulator; $l_{1}$ and $l_{2}$ are the arm lengths, respectively. $m_{w}$ is the mass of each driving wheel. $I_{m}$ is the moment of inertia of each wheel and the motor about the wheel diameter.

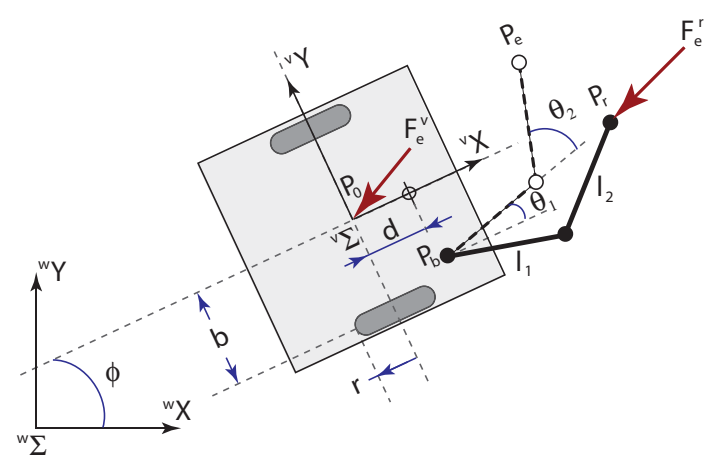

Fig. 1. Geometry of the mobile platform and the mounted $2 \mathrm{R}$ manipulator.

The mobile platform is adopted to have two co-axial wheels driven by motors. It is assumed that the manipulator itself is nonredundant, that is, the degree of freedom of the manipulator is less than or equal to six. The constraint equations to which the platform is subjected are given in matrix form as $A\left(q_{v}\right) \dot{q}_{v}=0$, where $q_{v}=\left[x_{0}, y_{0}, \theta_{r}, \theta_{l}\right]^{T}$ and $A\left(q_{v}\right)$ is given by

$$
A\left(q_{v}\right)=\left[\begin{array}{cccc}
-\sin \phi & \cos \phi & 0 & 0 \\
-\cos \phi & -\sin \phi & c b & c b
\end{array}\right]
$$

\section{B. State Space Formulation of Motion Equations}

The dynamics of the wheeled mobile manipulator are governed by motion Eqs. 3 and $A\left(q_{v}\right) \dot{q}_{v}=0$, we proceed to represent them in the state space. Since the platform velocity is always in the null space of $A\left(q_{v}\right)$ [4] according to $A\left(q_{v}\right) \dot{q}_{v}=0$, it is possible to define a vector of generalized coordinates $\eta(t)$ such that

$$
\dot{q}_{v}=S\left(q_{v}\right) \eta(t)
$$

where $S\left(q_{v}\right)$ is a $4 \times 2$ full rank matrix, whose columns are in the null space of $A\left(q_{v}\right) . S\left(q_{v}\right)$ is as follows [4]

$$
S\left(q_{v}\right)=\left[\begin{array}{cc}
c b \cos \phi & c b \cos \phi \\
c b \sin \phi & c b \sin \phi \\
1 & 0 \\
0 & 1
\end{array}\right] .
$$

First, we differentiate Eq. 5, and we substitute the resulting expression for $\ddot{q}_{v}$ into the first equation in Eqs. 3 which is multiplied by $S^{T}$, later, we proceed similarly using $\ddot{q}_{v}$ into the second equation in Eqs. 3. 
As a result, the system of equations is given by

$$
\begin{gathered}
\underbrace{\left[\begin{array}{cc}
S^{T} M_{v} S & S^{T} R_{v} \\
R_{r} S & M_{r}
\end{array}\right]}_{\xi}\left[\begin{array}{c}
\dot{\eta} \\
\ddot{q_{r}}
\end{array}\right]= \\
\underbrace{\left[\begin{array}{c}
-S^{T} M_{v} \dot{S} \eta-S^{T} C_{v} \\
-C_{r}-R_{r} \dot{S} \eta
\end{array}\right]}_{P}+\underbrace{\left[\begin{array}{cc}
S^{T} E_{v} & 0 \\
0 & I
\end{array}\right]}_{Q}\left[\begin{array}{c}
\tau_{v} \\
\tau_{r}
\end{array}\right] \\
+\underbrace{\left[\begin{array}{cc}
S^{T} E_{v} J_{v}{ }^{T} & 0 \\
0 & J_{r}^{T}
\end{array}\right]}_{D}\left[\begin{array}{c}
F_{e}{ }^{v} \\
F_{e}{ }^{r}
\end{array}\right] .
\end{gathered}
$$

Using the state vector $x=\left[\begin{array}{lll}q_{v}^{T} & q_{r}^{T} & \eta^{T}{\dot{q_{r}}}^{T}\end{array}\right]^{T}$, the system can be rewritten as

$$
\dot{x}=\underbrace{\left[\begin{array}{c}
S \eta \\
\dot{q}_{r} \\
P^{-1} \xi
\end{array}\right]}_{F(x)}+\underbrace{\left[\begin{array}{c}
0 \\
0 \\
P^{-1} Q
\end{array}\right]}_{G(x)} \tau+\underbrace{\left[\begin{array}{c}
0 \\
0 \\
P^{-1} D
\end{array}\right]}_{p(x)} \omega,
$$

where $\tau=\left[\begin{array}{ll}\tau_{v} & \tau_{r}\end{array}\right]^{T}$ and $\omega=\left[\begin{array}{ll}F_{e}{ }^{v} & F_{e}{ }^{r}\end{array}\right]^{T}$.

The state space form of the system is

$$
\dot{x}=F(x)+G(x) \tau+p(x) \omega
$$

\section{Feedback Control and Disturbance DECOUPLING}

We have followed the work of Yamamoto and Yun [4] in the derivation of the output equations, section A. Section B is the new work made for the disturbance decoupling problem.

\section{A. Output Equations}

A control system is fully described by the state equation and the output equation. The state equation of the mobile manipulator was obtained in the previous section. The output variables are the ones whose values are to be regulated by the design of a nonlinear feedback controller.

The desired task trajectory for the endpoint of the manipulator $P_{e}$ in the frame $\Sigma_{w}$ is given by

$$
{ }^{w} P_{e}(t)=\left[\begin{array}{l}
{ }^{w} x_{e}(t) \\
{ }^{w} y_{e}(t)
\end{array}\right] .
$$

The mobile manipulator shown in Fig. 1 has four inputs, two from the $2 \mathrm{R}$ manipulator and two from the mobile platform. We may have up to four output variables to be controlled. First, we select the output variables of the manipulator. $P_{e}$ represents the actual location of the end point of the manipulator. The coordinates of $P_{e}$ with respect to the platform coordinate frame $\Sigma_{v}$ are given by

$$
{ }^{v} P_{e}=\left[\begin{array}{l}
{ }^{v} x_{e} \\
{ }^{v} y_{e}
\end{array}\right]=\left[\begin{array}{c}
l_{1} \cos \theta_{1}+l_{2} \cos \left(\theta_{1}+\theta_{2}\right)+{ }^{v} x_{b} \\
l_{1} \sin \theta_{1}+l_{2} \sin \left(\theta_{1}+\theta_{2}\right)+{ }^{v} y_{b}
\end{array}\right] .
$$

Points ${ }^{v} P_{e}$ and ${ }^{w} P_{e}$ are related by

$$
{ }^{w} P_{e}={ }^{w} P_{0}+R_{\phi}{ }^{v} P_{e}=\left[\begin{array}{l}
x_{0} \\
y_{0}
\end{array}\right]+\left[\begin{array}{cc}
\cos \phi & -\sin \phi \\
\sin \phi & \cos \phi
\end{array}\right]\left[\begin{array}{l}
{ }^{v} x_{e} \\
{ }^{v} y_{e}
\end{array}\right] .
$$

The output variables for controlling the mobile platform are chosen next. The objective of the platform movement is to bring the manipulator into a preferred configuration. For this purpose, we pick the configuration with the maximum manipulability measure as the preferred configuration of the manipulator. The endpoint of the manipulator at the preferred configuration is denoted by $P_{r}$, called the reference point. The coordinates of $P_{r}$ in $\Sigma_{v}$ are given by

$$
{ }^{v} P_{r}=\left[\begin{array}{c}
{ }^{v} x_{r} \\
{ }^{v} y_{r}
\end{array}\right]=\left[\begin{array}{c}
\sqrt{l_{1}{ }^{2}+l_{2}{ }^{2}}+{ }^{v} x_{v} \\
{ }^{v} y_{b}
\end{array}\right]=\left[\begin{array}{c}
l_{x} \\
l_{y}
\end{array}\right] .
$$

We look to control the mobile platform in such a way that $P_{r}$ is brought to $P_{e}$, so the manipulator is brought into the preferred configuration. Thus, we select the coordinates of $P_{r}$ in the inertial frame $\Sigma_{w}$, i.e.

$$
{ }^{w} P_{r}=\left[\begin{array}{l}
{ }^{w} x_{r} \\
{ }^{w} y_{r}
\end{array}\right]=\left[\begin{array}{l}
x_{0} \\
y_{0}
\end{array}\right]+\left[\begin{array}{cc}
\cos \phi & -\sin \phi \\
\sin \phi & \cos \phi
\end{array}\right]\left[\begin{array}{l}
l_{x} \\
l_{y}
\end{array}\right] .
$$

to be the other two components of the output equation. The output equations for controlling the mobile manipulator are given by

$$
y=\underbrace{\left[\begin{array}{c}
{ }^{w} x_{r}\left(x_{0}, y_{0}, \theta_{r}, \theta_{l}\right) \\
{ }^{w} y_{r}\left(x_{0}, y_{0}, \theta_{r}, \theta_{l}\right) \\
{ }^{v} x_{e}\left(\theta_{1}, \theta_{2}\right) \\
{ }^{v} y_{e}\left(\theta_{1}, \theta_{2}\right)
\end{array}\right]}_{h(x)} .
$$

\section{B. Feedback Input-Output Linearization with Disturbance De- coupling}

We have presented the dynamics of the mobile manipulator in the state space form and the output equation

$$
\begin{aligned}
\dot{x} & =F(x)+G(x) \tau+p(x) \omega, \\
y & =h(x) .
\end{aligned}
$$

Note that the vector field $p(x)$ models the disturbances. It has been shown that this system is nonholonomic [4] and it is not input-output linearizable as it is. Thus, to achieve inputoutput linearization a nonlinear feedback has to be employed. To simplify state Eq. 9 we applied the following feedback,

$$
\tau=Q^{-1}(P u-\xi) .
$$

which simplifies the state equation as

$$
\begin{aligned}
\dot{x} & =\underbrace{\left[\begin{array}{c}
S \eta \\
\dot{q}_{r} \\
0
\end{array}\right]}_{f(x)}+\underbrace{\left[\begin{array}{c}
0 \\
0 \\
I
\end{array}\right]}_{g} u+\underbrace{\left[\begin{array}{c}
0 \\
0 \\
P^{-1} D
\end{array}\right]}_{p(x)} \omega, \\
y & =h(x) .
\end{aligned}
$$

If the disturbance $\omega$ is available for measurements one can use a control $u=\alpha(x)+\beta(x) v+\gamma(x) \omega$ [8]. Then decoupling the output from the disturbance it is possible. 
The relative degree of the system is $r=2$, that is the number of differentiations of each component of the outputs until the input explicitly appears in the derivative $\ddot{y}$. Following the analysis of [8], the control law solving the problem of decoupling $y$ from $\omega$ is given by

$$
\begin{aligned}
& \alpha(x)=-\frac{L_{f}^{2} h(x)}{L_{g} L_{f} h(x)}=-\frac{\dot{\Phi}}{\Phi}\left[\begin{array}{c}
\eta \\
\dot{q}_{r}
\end{array}\right], \\
& \beta(x)=\frac{1}{L_{g} L_{f} h(x)}=\frac{1}{\Phi}, \\
& \gamma(x)=-\frac{L_{p} L_{f} h(x)}{L_{g} L_{f} h(x)}=-P^{-1} D .
\end{aligned}
$$

The nonlinear feedback is given by

$$
u=\Phi^{-1}\left(v-\dot{\Phi}\left[\begin{array}{c}
\eta \\
\dot{q}_{r}
\end{array}\right]-\Phi P^{-1} D \omega\right) .
$$

The matrix $\Phi$ is presented in the Appendix. Applying this nonlinear feedback Eq. 13 into Eq. 12, we obtain a linear and decoupled input-output relationship

$$
\ddot{y}=\left[\begin{array}{l}
\ddot{y}_{1} \\
\ddot{y}_{2} \\
\ddot{y}_{3} \\
\ddot{y}_{4}
\end{array}\right]=\left[\begin{array}{l}
v_{1} \\
v_{2} \\
v_{3} \\
v_{4}
\end{array}\right]=v .
$$

The input-output relationship is decoupled because each component of the reference input, $v_{i}$, controls one and only one component of the output $y_{i}$. To complete the controller design, it is necessary to stabilize each of the above four subsystem with another constant feedback. Therefore, the entire controller for the mobile manipulator consists of nonlinear feedbacks Eq. 11 and Eq. 13, followed by a linear feedback. We have used a PD computed-torque control law. We look for a desired trajectory $y_{d}$, which gives $\ddot{y}=\ddot{y}_{d}-K_{v} \dot{e}-K_{p} e$ with the tracking error defined as $e=y-y_{d}$.

\section{Simulations}

Computer simulations are conducted to evaluate the effectiveness of the controller. In the simulation, a task trajectory will be examined. The mobile platform is initially placed at the origin facing toward the positive $X$-axis of the inertial frame. The initial head angle is zero, $\phi(0)=0$. The initial values $(t=$ $0)$ of the variables are $\left(x_{0}, y_{0}, \theta_{r}, \theta_{l}, \theta_{1}, \theta_{2}, \dot{\theta}_{r}, \dot{\theta}_{l}, \dot{\theta}_{1}, \dot{\theta}_{2}\right)=$ $(0,-0.15,0,0,45,-15,0,0,0,0)$. Platform and manipulator parameter values are given in Table I, we have used the values used in [4]. The entire system is assumed to be stationary at $t=0$.

We have selected a linear task trajectory

$$
{ }^{w} P_{e}(t)=\left[\begin{array}{l}
{ }^{w} x_{e}(t) \\
{ }^{w} y_{e}(t)
\end{array}\right]=\left[\begin{array}{l}
{ }^{w} x_{e}(0)+\frac{t}{2} \\
{ }^{w} y_{e}(0)+\frac{t}{2}
\end{array}\right],
$$

where $\left({ }^{w} x_{e}(0),{ }^{w} y_{e}(0)\right)=(0.6,0)$ are the coordinates of the manipulator end point in the inertial frame at the initial configuration. ${ }^{v} x_{b}=0.01 \mathrm{~m}$ and ${ }^{v} y_{b}=-0.01 \mathrm{~m}$.

The external force disturbance has been implemented as $F_{e}{ }^{v}(t)=\left[\begin{array}{ll}100 & 100\end{array}\right]^{T}$ and $F_{e}^{r}(t)=\left[\begin{array}{ll}100 & 100\end{array}\right]^{T}$ for $t_{1}<t<$ $t_{2}$.
TABLE I

PARAMETERS VALUES USED FOR THE SIMULATIONS

\begin{tabular}{|c|c|c|}
\hline \hline Parameters & Values & Units \\
\hline \hline$r$ & 0.075 & $\mathrm{~m}$ \\
$b$ & 0.171 & $\mathrm{~m}$ \\
$l_{1}$ & 0.4 & $\mathrm{~m}$ \\
$l_{2}$ & 0.4 & $\mathrm{~m}$ \\
$m_{1}$ & 4 & $\mathrm{~kg}$ \\
$m_{2}$ & 4 & $\mathrm{~kg}$ \\
$m_{c}$ & 94 & $\mathrm{~kg}$ \\
$m_{w}$ & 5 & $\mathrm{~kg}$ \\
$I_{c}$ & 6.609 & $\mathrm{~kg} \cdot \mathrm{m}^{2}$ \\
$I_{m}$ & 0.135 & $\mathrm{~kg} \cdot \mathrm{m}^{2}$ \\
$I_{w}$ & 0.010 & $\mathrm{~kg} \cdot \mathrm{m}^{2}$ \\
$d$ & 0 & $\mathrm{~m}$ \\
\hline \hline
\end{tabular}

We have investigated the following situation:

- Simulation I. In this simulation, we have investigated the effect of a force disturbance at some interval $1<t<2$. The motion of the mobile manipulator is shown in Fig. 2. The cart geometry and its center (+) are shown in Fig. 2, the solid line represents the trajectory of the end-point of the manipulator and a dashed line is used for the desired task trajectory.

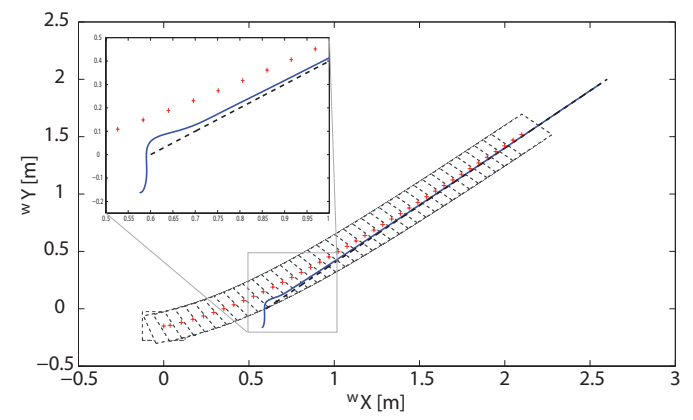

Fig. 2. Simulation I. Motion of the mobile platform during a linear task trajectory. Dashed line, linear task trajectory; Solid line, end-point of the manipulator;,$+ P_{0}$; dashed square; mobile platform position.

The variations of the joint angles of the manipulator during time are shown in Fig. 3. These angles have no significant changes during the simulation. The joint angles rate of change in time is shown Fig. 4.

The variation of the platform wheel angles during the simulation is shown in Fig. 5. There are slight changes at the initial stage, but later the angles are parallel to each other, which is reflected in the alignment of the axis of symmetry to the desired task trajectory. The platform wheel angles' rate of change is shown in Fig. 6. 


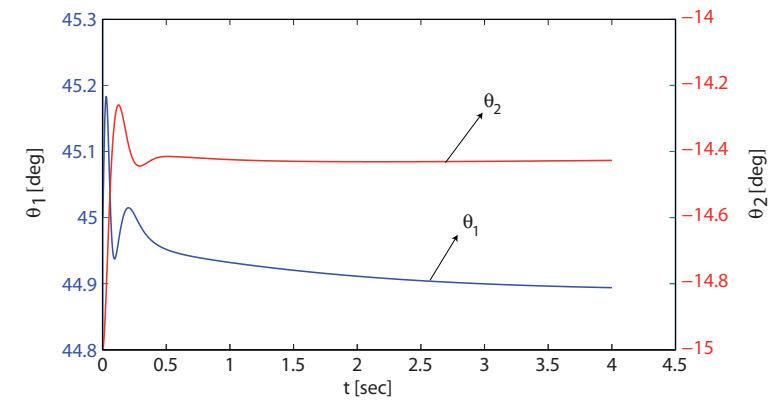

Fig. 3. Joint angles of the manipulator in time for Simulation I.

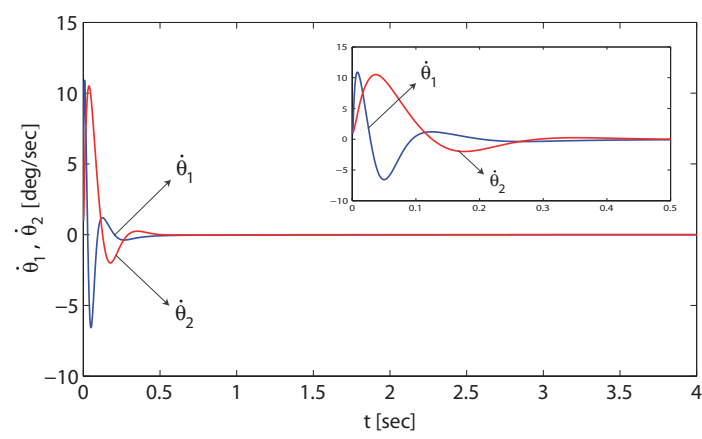

Fig. 4. Rate of change of the joint angles in time for Simulation I.

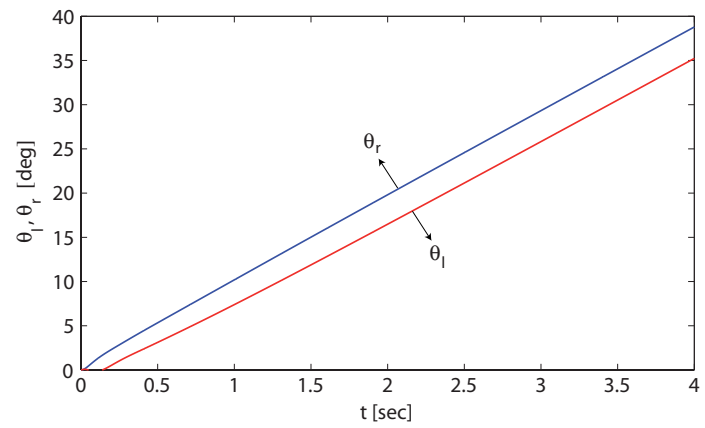

Fig. 5. Wheel angles of the mobile platform in time for Simulation I.

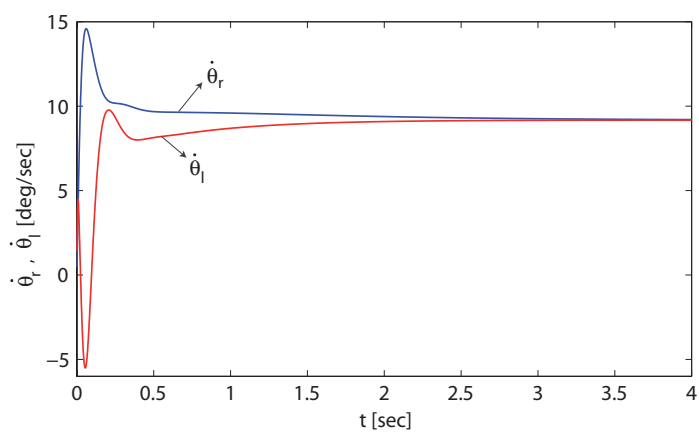

Fig. 6. Rate of change of the wheel angles in time for Simulation I.

The tracking errors are shown in Fig. 7. We have estimated the tracking error as the difference of the obtained trajectory to the desired trajectory as $e_{i}(t)=y_{i}(t)-y_{d_{i}}(t)$, for $i=$ $1, \ldots, 4$. During the simulation, initially there are oscillations in the tracking error, but later are reduced asymptotically to low values as expected.

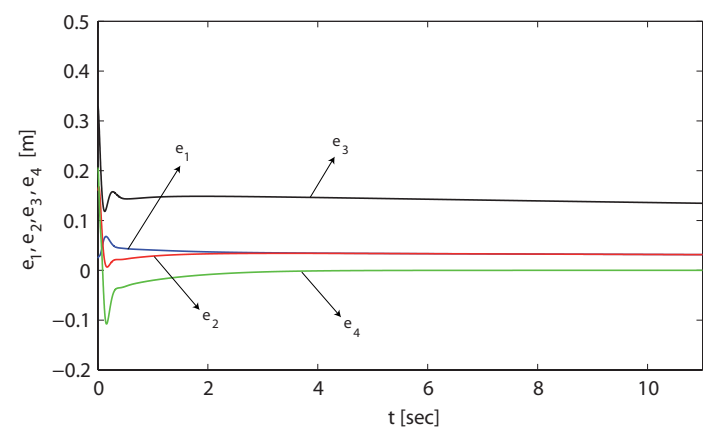

Fig. 7. Tracking errors for Simulation I.

The force disturbances are decoupled from the outputs. Consequently, the outputs do not change with the disturbances. The effect of force disturbance can be observed in the computed torques during the linear control. The computed torques for the simulation are shown in Fig. 8 and 9. We have compared the situation with disturbance $\left(F_{e}{ }^{v}(t)=\left[\begin{array}{ll}100 & 100\end{array}\right]^{T}\right.$ and $\left.F_{e}{ }^{r}(t)=\left[\begin{array}{ll}100 & 100\end{array}\right]^{T}\right)$ and without the disturbance.

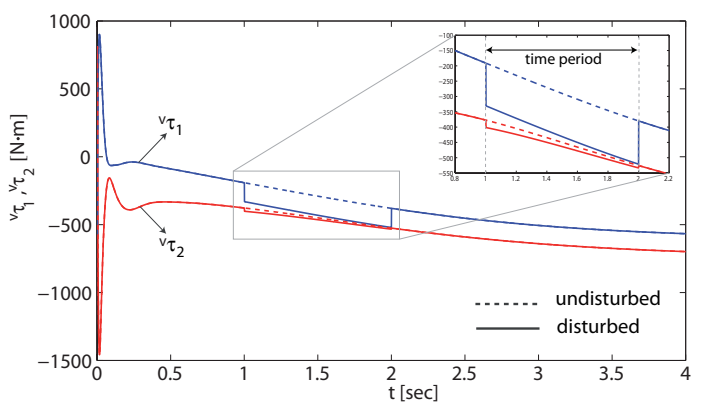

Fig. 8. Computed platform torques for Simulation I.

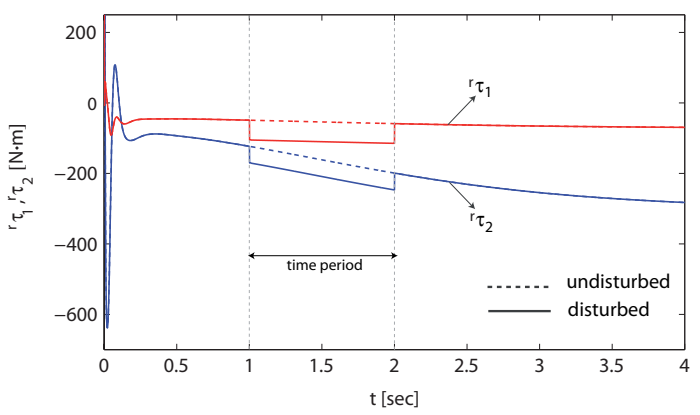

Fig. 9. Computed manipulator torques for Simulation I.

The disturbances are satisfactorily managed by the linear control applied to the linear input-output relationship.

\section{CONCLUSIONS}

We have presented the motion control of a mobile manipulator with disturbance decoupling of an external force.

First, we established a dynamic model which takes into 
account the dynamic interaction between the mobile platform and the mobile manipulator. We set up a nonlinear feedback controller law with disturbance decoupling and a linear control. This controller allows to the mobile platform to follow a desired trajectory even with disturbances. Finally, we conducted computer simulations, and the behavior of the variables of the problem was discussed.

\section{ACKNOWLEDGMENT}

The authors gratefully acknowledge the support of the U.S. Army and TARDEC under the SimBRS contract. UNCLASSIFIED: Dist A. Approved for public release.

\section{APPENDIX}

The following equations present the detailed expressions for all of the terms contained in the equations of motion for the system in this paper.

$$
\begin{aligned}
& q_{v}=\left[\begin{array}{lll}
{ }^{v} q_{1} & { }^{v} q_{2}{ }^{v} q_{3}{ }^{v} q_{4}
\end{array}\right]^{T}=\left[\begin{array}{llll}
x_{0} & y_{0} & \theta_{r} & \theta_{l}
\end{array}\right]^{T} \\
& q_{r}=\left[{ }^{r} q_{1}{ }^{r} q_{2}\right]^{T}=\left[\theta_{1} \theta_{2}\right]^{T} \\
& M_{r}=\left[\begin{array}{cc}
\frac{1}{3} m_{1} l_{1}^{2}+\frac{4}{3} m_{2} l_{2}{ }^{2}+m_{2} l_{2}{ }^{2} \cos \theta_{2} & \frac{1}{3} m_{2} l_{2}{ }^{2}+m_{2} l_{2}{ }^{2} \cos \theta_{2} \\
\frac{1}{3} m_{2} l_{2}^{2}+\frac{1}{2} m_{2} l_{2}{ }^{2} \cos \theta_{2} & \frac{1}{3} l_{2}{ }^{2} m_{2}
\end{array}\right], \\
& C_{r 1}=\left[\begin{array}{c}
-\frac{1}{2} m_{2} l_{2}^{2} \dot{\theta}_{2}^{2} \sin \theta_{2}-m_{2} l_{2}^{2} \dot{\theta}_{1}^{2} \dot{\theta}_{2}^{2} \sin \theta_{2} \\
\frac{1}{2} m_{2} l_{2}^{2} \dot{\theta}_{1}^{2} \sin \theta_{2}
\end{array}\right], \\
& C_{r 2}{ }^{(i)}=2 \sum_{j=1}^{m} \sum_{k=1}^{n} \sum_{h=\max (i, k)}^{n} \operatorname{tr}\left[\frac{\partial T_{h}}{\partial^{r} q_{i}} J_{h} \frac{\partial^{2} T_{h}{ }^{T}}{\partial^{v} q_{j} \partial^{r} q_{k}}\right]{ }^{v} \dot{q}_{j} \cdot{ }^{r} \dot{q}_{k} \\
& +\sum_{j=1}^{m} \sum_{k=1}^{n} \sum_{h=i}^{n} \operatorname{tr}\left[\frac{\partial T_{h}}{\partial^{r} q_{i}} J_{h} \frac{\partial^{2} T_{h}{ }^{T}}{\partial^{v} q_{j} \partial^{v} q_{k}}\right]{ }^{v} \dot{q}_{j} \cdot{ }^{v} \dot{q}_{k}, \\
& R_{r}{ }^{(i j)}=\sum_{k=i}^{n} \operatorname{tr}\left[\frac{\partial T_{k}}{\partial^{r} q_{i}} J_{k} \frac{\partial T_{k}^{T}}{\partial^{v} q_{j}}\right], \quad 1 \leq i \leq n, \quad 1 \leq j \leq m, \\
& T_{i}=T_{v} A_{1}{ }^{0} A_{2}{ }^{1} \ldots A_{i}{ }^{i-1}, \quad i=1, \ldots, n \text {, } \\
& A_{1}{ }^{0}=\left[\begin{array}{cccc}
\cos \theta_{1} & -\sin \theta_{1} & 0 & l_{1} \cos \theta_{1} \\
\sin \theta_{1} & \cos \theta_{1} & 0 & l_{1} \sin \theta_{1} \\
0 & 0 & 1 & 0 \\
0 & 0 & 0 & 1
\end{array}\right] \text {, } \\
& A_{2}{ }^{1}=\left[\begin{array}{cccc}
\cos \theta_{2} & -\sin \theta_{2} & 0 & l_{2} \cos \theta_{2} \\
\sin \theta_{2} & \cos \theta_{2} & 0 & l_{2} \sin \theta_{2} \\
0 & 0 & 1 & 0 \\
0 & 0 & 0 & 1
\end{array}\right] \text {, } \\
& T_{v}=\left[\begin{array}{cccc}
\cos \phi & \sin \phi & 0 & x_{0} \\
-\sin \phi & \cos \phi & 0 & y_{0} \\
0 & 0 & 1 & 0 \\
0 & 0 & 0 & 1
\end{array}\right] \text {, } \\
& J_{1}=\left[\begin{array}{cccc}
\frac{1}{3} m_{1} l_{1}^{2} & 0 & 0 & -\frac{1}{2} m_{1} l_{1} \\
0 & 0 & 0 & 0 \\
0 & 0 & 0 & 0 \\
-\frac{1}{2} m_{1} l_{1} & 0 & 0 & m_{1}
\end{array}\right] \text {, } \\
& J_{r}=\left[\begin{array}{cc}
-l_{1} \sin \theta_{1}-l_{2} \sin \left(\theta_{1}+\theta_{2}\right) & -l_{2} \sin \left(\theta_{1}+\theta_{2}\right) \\
l_{1} \cos \theta_{1}+l_{2} \cos \left(\theta_{1}+\theta_{2}\right) & l_{2} \cos \left(\theta_{1}+\theta_{2}\right)
\end{array}\right] \text {, }
\end{aligned}
$$

$$
\begin{aligned}
& J_{2}=\left[\begin{array}{cccc}
\frac{1}{3} m_{2} l_{2}{ }^{2} & 0 & 0 & -\frac{1}{2} m_{2} l_{2} \\
0 & 0 & 0 & 0 \\
0 & 0 & 0 & 0 \\
-\frac{1}{2} m_{2} l_{2} & 0 & 0 & m_{2}
\end{array}\right], \\
& M_{v 1}=\left[\begin{array}{cccc}
m & 0 & -m_{c} c d \sin \phi & m_{c} c d \sin \phi \\
0 & m & m_{c} c d \cos \phi & m_{c} c d \cos \phi \\
-m_{c} c d \sin \phi & m_{c} c d \cos \phi & I c^{2}+I_{w} & -I c^{2} \\
m_{c} c d \sin \phi & -m_{c} c d \cos \phi & -I c^{2} & I c^{2}+I_{w}
\end{array}\right] \text {, } \\
& C_{v 1}=\left[\begin{array}{c}
-m_{c} d \dot{\phi}^{2} \cos \phi \\
-m_{c} d \dot{\phi}^{2} \sin \phi \\
0 \\
0
\end{array}\right] \\
& E_{v}=\left[\begin{array}{ll}
0 & 0 \\
0 & 0 \\
1 & 0 \\
0 & 1
\end{array}\right] \\
& M_{v 2}{ }^{(i j)}=\sum_{k=1}^{n} \operatorname{tr}\left[\frac{\partial T_{k}}{\partial^{v} q_{i}} J_{k} \frac{\partial T_{k}^{T}}{\partial^{v} q_{j}}\right], \quad 1 \leq i, \quad j \leq m, \\
& C_{v 2}{ }^{(i)}=2 \sum_{j=1}^{n} \sum_{k=1}^{m} \sum_{h=j}^{n} \operatorname{tr}\left[\frac{\partial T_{h}}{\partial^{v} q_{i}} J_{h} \frac{\partial^{2} T_{h}{ }^{T}}{\partial^{r} q_{j} \partial^{v} q_{k}}\right]{ }^{r} \dot{q}_{j} \cdot{ }^{v} \dot{q}_{k} \\
& +\sum_{j=1}^{n} \sum_{k=1}^{n} \sum_{h=\max (j, k)}^{n} \operatorname{tr}\left[\frac{\partial T_{h}}{\partial^{v} q_{i}} J_{h} \frac{\partial^{2} T_{h}{ }^{T}}{\partial^{r} q_{j} \partial^{r} q_{k}}\right]{ }^{r} \dot{q}_{j} \cdot{ }^{r} \dot{q}_{k}, \\
& R_{v}{ }^{(i j)}=\sum_{k=j}^{n} \operatorname{tr}\left[\frac{\partial T_{k}}{\partial^{v} q_{i}} J_{k} \frac{\partial T_{k}^{T}}{\partial^{r} q_{j}}\right], \quad 1 \leq i \leq m, \quad 1 \leq j \leq n, \\
& \Phi=\left[\begin{array}{cccc}
\Phi_{1,1} & \Phi_{1,2} & 0 & 0 \\
\Phi_{2,1} & \Phi_{2,2} & 0 & 0 \\
0 & 0 & \Phi_{3,3} & \Phi_{3,4} \\
0 & 0 & \Phi_{4,3} & \Phi_{4,4}
\end{array}\right], \\
& \Phi_{1,1}=\left(c b-l_{y} c\right) \cos \phi-l_{x} \sin \phi, \\
& \Phi_{1,2}=\left(c b+l_{y} c\right) \cos \phi+l_{x} \sin \phi, \\
& \Phi_{2,1}=\left(c b-l_{y} c\right) \sin \phi+l_{x} \cos \phi, \\
& \Phi_{2,2}=\left(c b+l_{y} c\right) \sin \phi-l_{x} \cos \phi, \\
& \Phi_{3,3}=-l_{1} \sin \theta_{1}-l_{2} \sin \left(\theta_{1}+\theta_{2}\right) \text {, } \\
& \Phi_{3,4}=-l_{2} \sin \left(\theta_{1}+\theta_{2}\right) \text {, } \\
& \Phi_{4,3}=l_{1} \cos \theta_{1}+l_{2} \cos \left(\theta_{1}+\theta_{2}\right) \text {, } \\
& \Phi_{4,4}=l_{2} \cos \left(\theta_{1}+\theta_{2}\right) \text {. }
\end{aligned}
$$

\section{REFERENCES}

[1] M. W. Chen and A. M. S. Zalzala. Dynamic modeling and genetic based trajectory generation for non-holonomic mobile manipulators. Control Engineering Practice, 5(1):39-48, 1997.

[2] C. Wang and V. Kumar. Velocity control of mobile manipulators. Proceedings IEEE Int. Robotics and Automation, 2:713-718, 1993.

[3] J. H. Chung and S. A. Velinsky. Modeling and control of a mobile manipulator. Robotica, 16:607-613, 1998.

[4] Y. Yamamoto and X. Yun. Effect of the dynamic interaction on coordinated control of mobile manipulators. IEEE Transac. on robotics and automation, 12(5):816-824, 1996.

[5] Y. Yamamoto and X. Yun. A modular approach to dynamic modeling of a class of mobile manipulators. IEEE International Journal of Robotics and Automation, 12(2):41-48, 1997.

[6] Mohammad Danesh, Farid Sheikholeslam, and Mehdi Keshmiri. External force disturbance rejection in robotic arms: An adaptive approach. IEICE Trans. Fundamentals, E88-A(10):2504-2513, 2005.

[7] Jagdish Joshi and Alan A. Desrochers. Modeling and control of a mobile robot subject to disturbances. IEEE International Conference on Robotics and Automation, 1:1508-1513, 1986.

[8] Alberto Isidori. Nonlinear Control Systems. Springer, 2002. 